\section{RMD Open}

Rheumatic \&

Musculoskeletal Diseases

To cite: Favero M, Giusti A, Geusens $\mathrm{P}$, et al. OsteoRheumatology: a new discipline?. RMD Open 2015;1:e000083.

doi:10.1136/rmdopen-2015000083

- Prepublication history for this paper is available online. To view these files please visit the journal online (http://dx.doi.org/10.1136/ rmdopen-2015-000083)

Received 18 February 2015 Revised 28 April 2015 Accepted 1 May 2015

For numbered affiliations see end of article.

Correspondence to Dr Gerolamo Bianchi; gerolamo_bianchi@tin.it

\title{
OsteoRheumatology: a new discipline?
}

\author{
Marta Favero, ${ }^{1,2}$ Andrea Giusti, ${ }^{3}$ Piet Geusens, ${ }^{4}$ Steven R Goldring, ${ }^{5}$ Willem Lems, ${ }^{6}$ \\ Georg Schett, ${ }^{7}$ Gerolamo Bianchi ${ }^{8}$
}

\section{ABSTRACT}

This review summarises recent evidence about the interaction between bone, the immune system and cartilage in disabling conditions such as osteoarthritis, rheumatoid arthritis and spondyloarthritis. These topics have been recently discussed at the 'OsteoRheumatology' conference held in Genoa in October 2014. The meeting, at its 10th edition, has been conceived to bring together distinguished international experts in the fields of rheumatic and metabolic bone diseases with the aim of discussing emerging knowledge regarding the role of the bone tissue in rheumatic diseases. Moreover, this review focuses on new treatments based on underlying the pathophysiological processes in rheumatic diseases. Although, a number of issues still remain to be clarified, it seems quite clear that in clinical practice, as well as in basic and translational research, there is a need for more knowledge of the interactions between the cartilage, the immune system and the bone. In this context, 'OsteoRheumatology' represents a potential new discipline providing a greater insight into this interplay, in order to face the multifactorial and complex issues underlying common and disabling rheumatic diseases.

\section{INTRODUCTION}

The basic idea underlying OsteoRheumatology is the importance of the crosstalk between inflammation, cartilage and subchondral bone in disabling conditions such as rheumatoid arthritis (RA) and osteoarthritis (OA). The growing interest on the topic led to significant progresses during the past 20 years, resulting from the continuous efforts of clinical and basic researchers worldwide.

Basic and clinical research focused on the interactions between the bone tissue, primarily the subchondral bone, the bone cells (osteoblasts, osteoclasts and osteocytes), the immune system and the cartilage in several rheumatological diseases, such as OA, spondyloarthritis ( $\mathrm{SpA}$ ) and RA. In this context, the bone damage as an early manifestation of arthritides, the systemic skeletal involvement in RA, the crucial role played by subchondral bone in the pathogenesis and progression of OA, the pathophysiology of glucocorticoid damage to the bone tissue and the potential beneficial effects of bisphosphonates and newly approved agents such as denosumab and biologics have been the matter of the study and debate in the among basic/clinical investigators in the past decade.

The growing awareness about the relevant interactions between the bone, the cartilage and the immune system in a number of rheumatic diseases encouraged the organisation of the first 'Bone Involvement in Arthritis' International Meeting (held in Venice in 2004), with the aims of bringing together distinguished international experts in the fields of rheumatic and metabolic bone diseases and discussing emerging evidence regarding the role of the bone tissue in rheumatic diseases. In 2011, the meeting changed its name to 'OsteoRheumatology', thus implying the necessity of giving a more clear definition to topics presented and issues raised.

This brief overview summarises the recent insights from the last two meetings, which took place in Genoa (Italy) during the years 2013 and 2014. The programme was organised by a scientific committee, chaired by Professor Gerolamo Bianchi.

\section{BONE INVOLVEMENT IN OA}

$\mathrm{OA}$ is the most common joint disorder and is the major cause of disability in the adult population. ${ }^{1}$ Although cartilage destruction is the hallmark of OA, it is now well established that $\mathrm{OA}$ is not only a disorder of cartilage homeostasis but a whole-joint disease involving all of the articular tissues, including the subchondral bone and synovial membrane. ${ }^{2}$ More recently, some authors have extended this concept claiming that systemic factors such as low grade systemic inflammation (metabolic syndrome) or oestrogen deficiency are also involved in OA pathogenesis. ${ }^{3-5}$ For example, in women, oestrogen-deficiency has a strong influence on subchondral bone integrity and metabolism. ${ }^{4}$ In addition, there is evidence that, oestrogen-deficient animals develop 
slight but relevant histological changes in the healthy knee cartilage $^{6}$ and osteoporosis aggravates OA progression and severity in the OA-induced model in rabbit. ${ }^{6} 7$

\section{THE ROLE OF SUBCHONDRAL BONE IN OA}

The typical skeletal changes of OA are increased cortical plate thickness, flattening and deformation of the articular contour, bone marrow lesions, decreased subchondral trabecular bone mass, tidemark duplication and osteophyte formation. ${ }^{8}$ These changes affect the biomechanical properties of the overlying joint cartilage and intertwined biological relationship. The presence of subchondral bone stiffness may alter its viscoelastic proprieties and produce a loss of subchondral bone shock absorbing capacity with extra mechanical load. ${ }^{9-11}$ Several biological events confirming the presence of increased subchondral bone remodelling have been described in $\mathrm{OA}$ using different techniques, from scintigraphy to biomarkers. During progression of $\mathrm{OA}$, subchondral turnover appears to be increased 20-fold compared to normal bone turnover. ${ }^{12} 13$ The increased subchondral bone remodelling in OA can give two different phenotypes: sclerosis or osteoporosis, and both conditions may coexist in the same patient. Uneven mechanical overload on the osteochondral junction can induce a sclerotic phenotype, meanwhile post-menopausal global osteoporosis or other factors such as 'stress shielding' or synovial inflammation can produce a subchondral bone osteoporosis phenotype. Despite increased bone volume fraction, subchondral bone in OA is hypomineralised and of inferior quality compare to normal bone tissue. ${ }^{14}{ }^{15} \mathrm{It}$ has been suggested that hypomineralisation of the collagen matrix of the OA subchondral bone is due to the presence of atypical osteoblasts with a peculiar phenotype which produce an abnormal homotrimeric type I collagen with low affinity for calcium. ${ }^{16} \mathrm{OA}$ osteoblasts produce increased levels of alkaline phosphatase, osteocalcin, interleukin (IL)-6, IL-8, PG (prostaglandin)-E2, transforming growth factor (TGF) $\beta$ and insulin growth factor-1 (IGF-1). The local accumulation of IGF-1, which is a potent stimulator of bone formation, contributes to the subchondral bone sclerosis in OA. ${ }^{17}$ While IL-6, PGE2 and receptor activator of nuclear factor $\kappa-\mathrm{B}$ ligand (RANKL) may be responsible for increased number of osteoclasts in $\mathrm{OA}$ and bone resorption.

Recent studies have identified the central role of osteocytes in producing skeletal adaptive changes in $\mathrm{OA}$ in response to mechanical load. Osteocytes function as the bone mechanosensor and regulate bone remodelling by production of molecules that control both osteoclast and osteoblast differentiation and activity. ${ }^{18}$ The pathway that is involved in the osteoblast regulatory process by the osteocytes is the Wnt $\beta$-catenin pathway. In animal models increasing loading downregulates sclerostin production in osteocytes resulting in increased bone formation. ${ }^{19} 20$ Sclerostin expression is decreased in sclerotic subchondral bone in patients with $\mathrm{OA}$ and increased Wnt signaling in subchondral bone contributes to increased bone formation. ${ }^{21} 22$ In contrast, unloaded osteocytes are able to produce RANKL, ${ }^{23}{ }^{24}$ which enhances bone resorption. The damage to the subchondral bone matrix, in addition to mechanical loading, modulates the activity of the osteocytes and this process likely accounts for the development of the bone marrow lesion. ${ }^{25}$ Chondrocytes can also contribute to subchondral bone remodelling by producing RANKL. RANKL has been shown to be expressed in chondrocytes obtained from patients with knee OA, especially in the deep layer, ${ }^{26}$ and in cartilage of instability-induced OA animal models. ${ }^{27}$ RANKL and inflammatory factors released by articular cartilage may contribute to subchondral bone deterioration through increased bone remodelling in OA. The interaction between subchondral bone and cartilage is under investigation. Interestingly, in an in vivo cyclical compression OA animal model, cartilage degradation corresponds to progressive thickening of subchondral cortical bone with development of osteoporotic changes in the trabecular bone demonstrating a cross-talk between these two tissues in OA. ${ }^{28}$ Sanchez et $a l^{17}$ have demonstrated in vitro that osteoblasts from sclerotic zones of subchondral OA through IL-6 production, downregulate, aggrecan synthesis and upregulate MMPs expression by chondrocytes. Microcracks identified at the bone/cartilage junction and blood vessels invasion allow the exchange of biochemical factors produce by osteoblasts between the bone and overlying cartilage. ${ }^{16}$ The angiogenesis at the osteochondral junction is associated with hypertrophic differentiation of chondrocytes, which synthesize high levels of vascular endothelial growth factor (VEGF) and bone sialoprotein, two factors promoting endothelial cell proliferation and migration. ${ }^{29}$ In addition to the cytokines that regulate bone formation, the interaction of osteoclasts with the bone substrate also provides a potent signal to induce the osteoclast differentiation. ${ }^{30}$ Mechanisms involving subchondral bone in OA are summarised in figure 1.

\section{NEW TREATMENTS IN OA}

The actual treatments recommended for OA include paracetamol, non-steroidal anti-inflammatory drugs (NSAIDs) and opioids. No disease-modifying drugs are available to date for this pathology. ${ }^{31}$ Treatment of OA is not only a question of targeting the right molecule but also it is important to identify the proper subset of patients to treat considering the different phases of the disease. Moreover, different joint tissues can be involved in OA pathogenesis, including the synovium, cartilage and subchondral bone and they can be targeted by different pharmaceutical agent. Presently, OA is regarded as a heterogeneous disease, with different phenotypes. As a consequence, the next step is to investigate new treatment options in clearly defined clinical subsets within the broad OA population.

\section{Targeting pain}

Pain is the hallmark symptom of $\mathrm{OA}$ and the major reason for total joint replacement. Pain after inactivity, stiffness 
Figure 1 Different mechanisms may produce different phenotypes in osteoarthritis subchondral bone via increased subchondral bone remodelling. The figure summarises the role played by sclerostin, Wnt pathway, receptor activator of nuclear factor $\kappa-B$ ligand (RANKL) and other inflammatory cytokines in the pathogenesis of the different osteoarthritis subchondral bone phenotypes.
Mechanical overload on

osteochondral junction

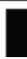

"Stress shielding" or

synovial inflammation

\section{Increased subchondral bone remodelling}
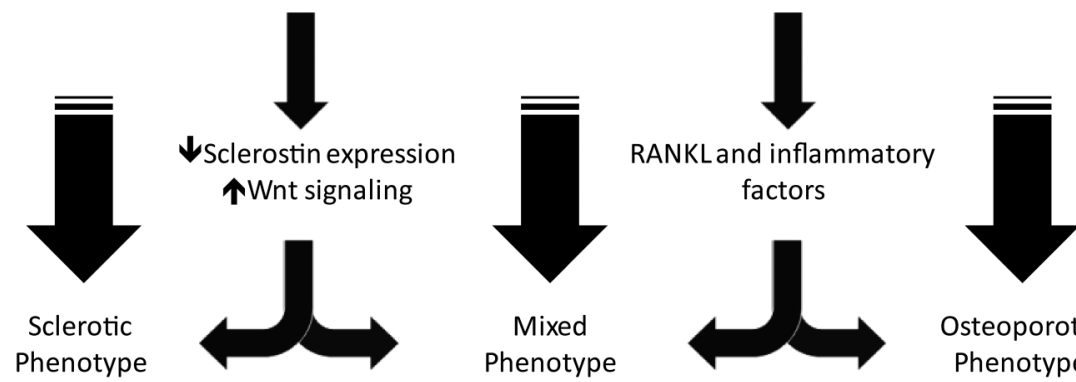

Osteoporotic Phenotype

and use-related pain are common, but rest and night pain particularly occur in severe OA. ${ }^{32}{ }^{33}$ Different patterns of pain have been identified connected with different stages of OA. In the early phases of the disease, pain is related to activity and becomes more constant over time. In the late stages there is 'background pain' interspersed with unpredictable intense pain, which highly impacts the quality of life and the participation in social and recreational activities. ${ }^{34}$ Sometimes, pain is more severe than other times, probably related to inflammation.

Cartilage is not innervated, but other joint tissues can be a source of pain such as the meniscus, synovium, capsule, ligaments and periosteum. Moreover, in severe OA there is vascular and neural invasion at the bone-cartilage junction. An understanding of the pathophysiological mechanisms of pain in $\mathrm{OA}$ is essential to developing new therapeutic targets. ${ }^{35}$ Activation of nociceptive pathways is a physiological response to tissue injury and damage, and it is vital for the organism. ${ }^{36} \mathrm{In}$ the presence of joint inflammation and associated with mechanical injury in patients with $\mathrm{OA}$, the release of inflammatory mediators such as cytokines ${ }^{37}$ can induce a peripheral sensitisation and lead to a decrease in the excitation threshold (allodynia=normally innocuous stimuli may evoke a pain response) or an increase in responsiveness to supra-threshold stimuli of peripheral nociceptors (hyperalgesia=noxious mechanical stimuli can evoke exaggerated response). Corticosteroids and NSAIDs act by inhibiting prostaglandins, which are proinflammatory mediators involved in peripheral sensitisation. Other than peripheral sensitisation there also is evidence that central sensitisation is involved in the pathogenesis of pain in OA. ${ }^{38}$ New therapeutic molecules can target the nociceptors to attenuate the peripheral sensitisation phenomenon, which characterises OA. Nerve grow factor (NGF) has been detected in the OA vascular channels at the osteochondral junction, and osteochondral angiogenesis is associated with increased NGF expression. This may facilitate the sensitisation or the invasion into the articular cartilage of sensory nerves. ${ }^{39}$ The expression of substance $\mathrm{P}$ and calcitonin gene-related peptide (CGRP) are increased by NGF. Different anti-NGF molecules are under investigation for the treatment of $\mathrm{OA}$ pain. The clinical trials with anti-NGF drugs, such as tanuzemab, have demonstrated a significant reduction in knee pain compared with the control population. ${ }^{40}{ }^{41}$ Nevertheless, in 2010 the Food and Drug Administration (FDA) suspended clinical trials because of cases of rapidly progressive $\mathrm{OA}$ in some patients. After reviewing of all the cases associated with anti-NGF treatment and concomitant use of NSAIDs, in 2012 the FDA recommended reinitiating trials with an alert of avoiding coprescription of NSAIDs. ${ }^{42}$

CGRP is another potential and interesting molecule for treating OA pain. CGRP acts through the calcitonin receptor-like receptor (CLRL) and the cofactor RAMP1. There is evidence indicating an association between joint levels of CGRP and pain during OA. ${ }^{43}{ }^{44}$ CGRP has been found colocalised with NGF in the vascular channels at the osteochondral junction in $\mathrm{OA}^{39}$ and it seems to be implicated in peripheral sensitisation in experimental OA. ${ }^{45}$ Moreover, CGRP is expressed in the meniscus ${ }^{46}$ and synovium ${ }^{47}$ and its expression correlates with chondral degeneration. Small molecule CGRP receptor antagonists have been recently developed and their efficacy in treating migraine pain in human subjects and OA pain in the monosodium iodoacetate animal model has been demonstrated. ${ }^{48}$ These findings indicate that CGRP represents an important neuropeptide that can be targeted in the treatment of OA pain. The presence of pain has been shown to correlate with MRI findings of moderate or large effusions, as well synovial thickening. ${ }^{49-51}$ In addition, pain has been demonstrated to correlate with synovitis in patients undergoing meniscectomy without radiological signs of OA. ${ }^{52} \mathrm{OA}$ pain has been associated with bone marrow lesions detected on MRI and changes in bone marrow oedema have been correlated with fluctuation in OA knee pain. ${ }^{50}{ }^{53}$ According to these observations, targeting subchondral bone and synovial inflammation may be reasonable therapeutic targets to address pain pathogenesis in OA, and this will be discussed in the next paragraphs. 


\section{Targeting subchondral bone}

Bone marrow lesions identified by MRI in patients with $\mathrm{OA}$ are usually poorly circumscribed areas seen on fat suppressed images that occur in weight-bearing areas just underneath the cortical bone. Histologically, they are characterised by the presence of microfractures, increased osteoclasts resorption and woven bone. ${ }^{54}$ Bisphosphonates are antiresorptive agents that mainly act on osteoclasts. The major rationale for using these drugs to treat $\mathrm{OA}$ pain is related to the abnormal bone turnover in $\mathrm{OA}$, which leads to a zone of osteoporosis beneath the subchondral plate, which results in decreased bone mechanical properties predisposing to bone microfractures. ${ }^{8}$ Osteoclasts contribute to vascular channel formation at the osteochondral junction resulting in vascular and neural invasion of the calcified articular cartilage and exposing nerves to proinflammatory mediators from the synovial fluid. ${ }^{39}$ Furthermore, osteoclasts may reduce $\mathrm{PH}$ at the osteochondral junction resulting in a sensitisation of the sensory nerves. ${ }^{55}$ A recent published review of the use of bisphosphonates for management of $\mathrm{OA}$ pain concludes that there is limited evidence that biphosphonates are effective in treatment of $\mathrm{OA}$ pain. ${ }^{56}$ Nevertheless, some limitations such as different duration of the treatment, dose, route of administration, lack of long-term data on OA joint structure modification have been highlighted. ${ }^{56}$ The efficacy of bisphosphonates in OA is controversial and some authors hypothesize that only the osteoporotic subchondral bone phenotype may benefit from antiresorptive treatment by decreasing bone remodelling and reducing bone resorption. ${ }^{15}{ }^{57}$ Lack of efficacy of bisphosphonates may be explained by the limited potency of some molecules and by the timing of treatment in a heterogeneous diseases with different phases. Moreover, structural disease modification is measured in OA mainly by joint space narrowing, which is influenced by joint tissues that may not be a source of pain. In the literature, pain in OA has been associated with bone marrow lesions and synovitis. Interestingly, a recent study has shown that Zoledronate, a potent bisphosphonate, significantly reduces pain and bone marrow lesions at 6 months in patients with OA and compared to controls. ${ }^{58}$ This study highlights the concept of using the right treatment at the right time. Subcutaneous tiludronate has been showed to have an effect in a OA model of dogs in reducing not only pain and increasing subchondral thickness but also synovitis. ${ }^{59}$ This data are consistent with the effect of bisphosphonates on subchondral bone turnover but also suggests a possible action on synovitis. ${ }^{60}$

Strontium ranelate has recently demonstrated to have a structure modifying effect (evaluated semiautomatically by joint space narrowing) at both 1 and $2 \mathrm{~g}$ doses and a beneficial effect on pain at $2 \mathrm{~g}$ /day (but not with $1 \mathrm{~g} /$ day) compared with placebo over 3 years of treatment. $^{61} 62$ Importantly, strontium ranelate has been shown to reduce cartilage volume loss in the tibial plateau and bone marrow lesion progression in the medial knee compartment as assessed by MRI. ${ }^{63}$ Its further development is hampered by side effects, such as cardiovascular events. ${ }^{64}$ Nevertheless, the above described SEKOIA study ${ }^{61}{ }^{62}$ is a landmark study, since it showed that the reduction of radiological joint damage was possible with the use of an antiosteoporotic drug.

\section{Targeting inflammation}

Unlike in RA, it has been demonstrated that blocking IL-1 $\beta$ in OA synovium did not result in inhibiting production of tumour necrosis factor $\alpha(\mathrm{TNF}-\alpha)$. In addition, treatment of osteoarthritic synovium with anti-TNF $\alpha$ agents did not block IL- $1 \beta .{ }^{65}$ Anti-TNF- $\alpha$ has not been shown to be effective in preventing the erosive structural damage of patients affected by erosive hand $\mathrm{OA}^{66}$ while $70 \%$ of patients with knee $\mathrm{OA}(\mathrm{N}=20)$ achieved an OARSI/OMERACT response in an openlabel evaluation of adalimumab over 12 weeks. ${ }^{67}$ Blocking iNOS, which is a proinflammatory mediator in $\mathrm{OA}$, failed to slow down or decrease OA progression in a 2-year randomised, double-blind, placebo-controlled, multicentre study of an oral selective iNOS inhibitor in patients with symptomatic OA of the knee. ${ }^{68}$ Even treatments with a single intra-articular injection of IL-1 receptor antagonist (IL-1Ra) ${ }^{69}$ and subcutaneous injections every 4 weeks for 3 months with an anti-IL-1 RI monoclonal antibody, which inhibits both IL-1 $\alpha$ and IL-1 $\beta$, has not shown a difference in terms of WOMAC scores in treated patients compared with placebo in knee $\mathrm{OA}^{70}$ Interestingly, preoperative intra-articular injection of IL-1Ra significantly reduced pain and synovial fluid cytokine levels in a small study $(\mathrm{N}=11)$ in patients undergoing ACL reconstruction for ACL rupture. $^{71}{ }^{72}$ Since ACL rupture has been correlated with the development of $\mathrm{OA}$, this data suggests that anti-IL-1 $\beta$ may be effective in the early phases of the disease, highlighting the concept that is important to identify distinct subsets of patients for treatment interventions.

\section{BONE INVOLVEMENT IN RA}

The physiological bone remodelling cycle includes activation of osteoclasts from precursors, resorption, reversal phase and bone formation mediated by osteoblasts. This system permits individuals to adapt the skeleton to mechanical forces, to repair damage and contributes to calcium and phosphorus mineral ion homeostasis. During adulthood, prior to the arrival of menopause and the effects of aging, the resorptive and formation processes are intimately coupled in a state of equilibrium. In the inflammatory joint diseases this balance between bone catabolism and anabolism is lost. Understanding the physiology of this process provides insight for therapeutic interventions. The major mechanism of 'coupling' bone remodelling after the resorption phase includes the release of growth factors trapped in the bone matrix, which initiates the phase of bone 
formation. This provides a mechanism for quantitatively linking bone resorption and the initiation of bone formation.

\section{BONE EROSIONS IN RA}

Osteoclasts are the essential cells that mediate the resorptive process under physiological conditions. In RA cells with phenotypic features of osteoclasts are present in resorption lacunae at the bone synovial interface. ${ }^{73}$ The pannus and inflammatory synovium are a very rich source of myeloid precursors and immunomodulatory and proinflammatory factors with osteoclastogenic activity. ${ }^{74-78}$ Indeed, macrophage lineage cells can differentiate into the osteoclasts, induced by factors produced by RA synovium, in particular RANKL and macrophage colony-stimulating factor (M-CSF). Of interest, the synovium adjacent to the resorption sites has been shown to be an abundant source of RANKL. ${ }^{79}$ It has been demonstrated that blocking osteoclasts represents a rational approach to prevent bone resorption in RA. ${ }^{80}$ RANKL knock-out (KO) mice lack osteoclasts and develop a severe form of osteopetrosis. To define the role of osteoclasts in the pathogenesis of bone erosions, serum from arthritic $\mathrm{K} / \mathrm{BxN}$ mice containing pathogenic antiglucose-6-phosphate isomerase antibodies was transferred to RANKL gene KO mouse resulting in the development of arthritis that histologically resembles RA. In the absence of osteoclasts, despite marked synovial inflammation and pannus formation, the RANKL KO mice do not develop significant bone erosions compared to control mice. ${ }^{80}$ Similar results have been obtained in two other RA animal models lacking osteoclasts. ${ }^{81} 82$ This highlights the concept that osteoclasts play an essential role in the pathogenesis of focal bone erosions in RA.

In RA in addition to an increased bone resorption there is a decrease of bone formation at the erosion sites in active RA, indicating the presence of uncoupling

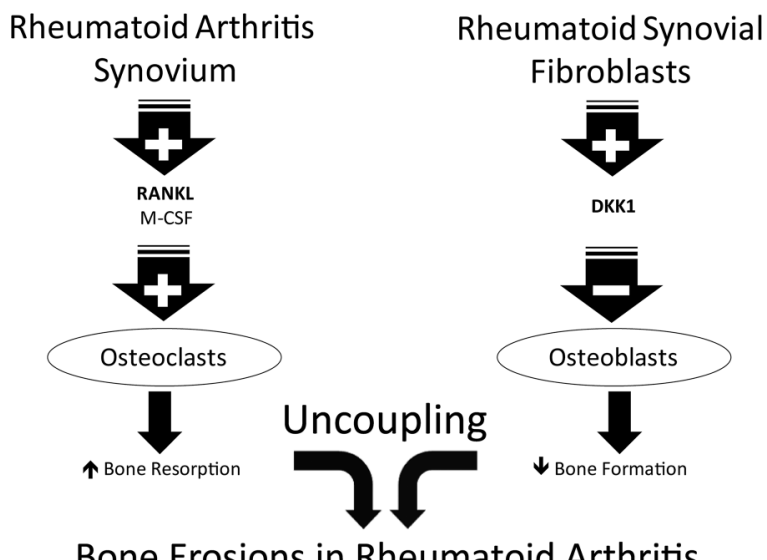

Figure 2 Uncoupled bone remodelling and bone erosion formation in rheumatoid arthritis. In rheumatoid arthritis patients an increased bone resorption and a decreased bone formation lead to bone erosion formation. of bone remodelling. Of interest when the joint inflammation is reduced by treatment there is partial restoration of bone formation. ${ }^{83}$

Regulation of osteoblast differentiation involves both Wnt ligands and BMPs as inducers of bone formation. Sclerostin and DKK-1 are inhibitors of the classical canonical Wnt/ $\beta$-Catenin signaling pathway and they block the interactions between the receptor complex consisting of LRP5/ 6 and frizzled and Wnt resulting in a degradation of $\beta$-catenin and inhibition of bone formation. ${ }^{84}$ Antisclerostin antibodies have been demonstrated to increase bone mass in animal models of osteoporosis and fracture repair. ${ }^{85-87}$ Rheumatoid synovial fibroblasts are a very rich source of $D K K 1^{88}$ and TNF- $\alpha$ is an important inducer of DKK-1. At least in part, the uncoupling of bone formation and the lack of bone repair in the rheumatoid process could be attributed to the production in the synovial tissue of this inhibitor of the Wnt pathway. Mechanisms leading to bone erosions in RA are summarised in figure 2.

\section{SYSTEMIC BONE LOSS IN RA}

In the pathogenesis of RA, two phases have been recognised: (1) an autoimmune phase, induced by environmental factors such as smoking, acting in a certain genetic background that imparts susceptibility. During this autoimmune phase, patients may develop rheumatoid factor (RF) and anticitrullinated protein antibodies (ACPA) over 10 years before the clinical manifestations of the disease. ${ }^{89-91}$ (2) an inflammatory phase, possibly induced by an infection or other environmental triggers, which leads to a chronic inflammatory state, which can ultimately affect multiple organs, including the bone. ${ }^{92}$ Both phases can induce bone alterations. Bone is one of the key organ targets in RA. In the literature there are many examples of an increased fracture, including vertebral and non-vertebral and, hip. Fracture risk in patients with RA is due to not only to trabecular but also cortical bone loss. ${ }^{93-98}$

Bone structure alterations, including reduced bone volume and bone mineral density (BMD), cortical bone thickness and increased fenestration have been seen in ACPA positive non-arthritic individuals compared to ACPA-negative controls. ${ }^{99}$ There are several observations supporting these data and demonstrating that bone metabolism is altered before the onset of the disease. ${ }^{100-102}$ Moreover, ACPA-positive patients are at risk for developing severe bone erosions with extensive resorption of the trabecular network. ACPAs isolated from sera of patients with RA have been shown to induce osteoclastogenesis and bone resorption in vitro. ${ }^{103}$ In addition, ACPA injections in immunodeficiency mice induce bone loss after 4 weeks due to increased osteoclast formation. ${ }^{104}$ ACPAs can induce osteoclast activation and bone loss starting 10 years before the onset of the clinical phase of the disease, prior to a second hit, which is associated with the appearance of overt synovitis. ${ }^{99}$ It has been suggested that the preclinical 
phase of RA primes the joint to the inflammatory phase because cortical fenestration induced by osteoclastic activity lead to a communication between bone marrow and synovial tissue. Bone marrow oedema in subchondral bone in RA predicts the development of bone erosions and there is evidence suggesting than plasma cells can trigger osteoclastogenesis by local production of antibodies. ${ }^{105}$ The synovial inflammation induces bone loss triggering an imbalance between bone resorption and formation similar to what occurs with some tumours. ${ }^{106}$ In an inflamed joint, the resorptive process immediately starts with the onset of the disease. Osteoclast precursors and osteoclasts has been found respectively after 2 and 5 days in the collagen-induced arthritis mouse model. ${ }^{107}$ The synovial inflammatory tissue instruct T cells to produce M-CSF and RANKL which induce osteoclastogenesis. ${ }^{108}$

\section{NEW TREATMENTS TARGETING BONE IN RA}

RANKL is highly expressed in the synovial membrane of patients with RA leading to osteoclast differentiation and activation. ${ }^{109} 110$ Treatment with denosumab, a human RANKL monoclonal antibody, increases lumbar spine BMDand reduces vertebral and non-vertebral fracture rates in postmenopausal women with osteoporosis, ${ }^{111}$ but also protects patients with RA from bone erosions, by arresting osteoclast formation and activation. ${ }^{112} 113$ Nevertheless denosumab does not have an effect on inflammation and its use would need to be combined with an anti-inflammatory strategy. Many cytokines including TNF- $\alpha$, IL-1 $\beta$, IL- 6 are involved in the osteoclast activation process leading to bone loss in patients with RA. ${ }^{114}$ It has been demonstrated that TNF- $\alpha$ is a very potent inducer of osteoclasts. ${ }^{115}{ }^{116}$ So the inhibitions of these cytokines can block bone erosions in addition to inhibitory effects on inflammation. IL-17 is one of the major drivers of RANKL production by synovial fibroblasts and is a potential target for the treatment of inflammatory arthritis and associated bone disease. As discussed above, TNF- $\alpha$ is able to block bone formation by production of inhibitors of osteoblast differentiation such as Dkk1 and sclerostin, in addition to its ability to enhance osteoclast differentiation..$^{88117118}$

\section{BONE INVOLVEMENT IN SpAs}

The SpAs are a heterogeneous group of chronic inflammatory diseases of the skeleton and associated soft tissues, including ankylosing spondylitis (AS), psoriasic arthritis (PsA), inflammatory bowel disease-associated arthritis (IBD), reactive arthritis, juvenile SpA and undifferentiated SpA. All subtypes of SpA are characterised by inflammation and structural damage of the axial and/or peripheral skeleton. The inflammatory process presents as osteitis, enthesitis, synovitis and may produce joint destruction with loss of articular cartilage and bone erosion. On the other hand, and in contrast to RA, skeletal abnormalities are manifest as new bone formation leading to bony fusion of the sacroiliac joints, syndesmophyte formation (with bridging of the intervertebral spaces) and enthesophyte formation. These processes, that are the hallmarks of the SpAs, contribute significantly to permanent disability and loss of function of the patients. Skeletal growth during development and growth, occurs by two different mechanisms: endochondral bone formation, by which the skeletal elements are defined by an intermediate cartilage stage; and membranous bone formation, that results from direct new bone formation. These processes rely on a number of molecular signaling pathways, that include bone morphogenic proteins (BMPs), Wnts, hedgehog, fibroblast growth factor, notch and parathyroid hormone-like peptide signaling. Although relatively few data are available for SpAs, animal models and human data suggests that both endochondral and intramembranous bone formation contribute to ankylosis in patients with SpA. ${ }^{119-121}$

A number of well-designed studies in animal models have highlighted the role of BMP and Wnt signaling in the process of ankylosis in SpA patients. Lories et al ${ }^{120}$ studied the spontaneous arthritis model in aging male DBA/1 mice to better understand the molecular mechanism of ankylosing enthesitis, and demonstrated that different BMPs are expressed during the process of ankylosis in this model. ${ }^{121}$ In the spontaneous ankylosing enthesitis model, the over-expression of noggin, a BMP antagonist, produced an inhibition of ankylosis. ${ }^{122}$ In another study, in the human TNF transgenic mouse model of arthritis, Diarra $e t a l^{88123}$ established the link between Wnt signaling and new bone formation. They studied the effect of blocking Dickkopf-1 (DKK1), an antagonist of Wnt signaling pathway. By blocking the DKK1, the phenotype of the disease shifted from joint destruction to joint remodelling with formation of osteophytes in the peripheral joints and ankylosis of the sacroiliac. The blockade of DKK1 function is associated with the activation of the canonical Wnt pathway and increased levels and nuclear translocation of $\beta$-catenin with resultant increased bone formation.

Human data further supported these animal observations. Analyses of pathological tissue from patients with SpA revealed the presence of BMPs and activation of BMP signaling in peripheral entheseal lesions. ${ }^{120}{ }^{121}$ In addition, Diarra $e t a l^{88}$ found that serum levels of DKK1 are very low or absent in patients with AS as compared to subjects with RA.

In conclusion, these and other experimental data strongly suggest that BMPs and Wnt signaling through $\beta$-catenin, play key roles in the pathogenesis of the enhanced periarticular bone formation and ankylosis in the SpAs. Current data, particularly in animal models, suggest that BMPs and Wnt pathways might represent potential targets for the prevention of ankylosis in SpA.

\section{NEW TREATMENTS IN SpAs}

The introduction of anti-TNF drugs produced significant and relevant success in the management of SpAs. Anti-TNF therapies are highly effective in treatment of pain and stiffness in patients affected by SpAs, but these drugs have not been shown to slow the radiographic 
progression in clinical trials. ${ }^{124}$ However, the development of radiographic changes in SpAs is slow and placebo-controlled clinical trials have been carried out at a maximum for 24 weeks for ethical reasons. Interestingly, two recent observational studies in patients with AS, respectively at 8 years and up to 6.3 years showed a potential effect of anti-TNF therapy on slowing radiographic progression other than reducing inflammation. ${ }^{125} 126$

In addition, high-intensity or continuous NSAIDs have been shown to affect the AS radiographic progression, respectively, in a 2 year open-label randomised clinical trial and in a retrospective observational study. Interpretation of these results is limited by the fact that the first study had an unblinded design, while the second had a potential selection bias.

There are two emerging biologics, primarily developed in psoriasis and PsA, which seem to be effective not only in reducing inflammation but also in preventing bone formation in AS: ustekinumab (UST), a compound targeting IL-12/IL-23 via the p40 subunit of both cytokines and the anti-IL-17 antibody secukinumab (SEC). T cells differentiated to the Th17 phenotype under the influence of IL-23 are characterised by the production of IL-17. IL-23 and IL-17 levels are increased in blood samples of patients with AS and detected in synovial biopsies of these patients. Moreover, IL-23 receptor polymorphisms are associated with susceptibility to SpAs. ${ }^{127}$ The primary structure involved in spondyloarthropathy is the enthesis, and IL-23 has been demonstrated to stimulate enthesitis and periosteal bone formation in a collagen antibody-induced animal model. Blocking IL-23 at the time of disease induction reduced clinical disease scores and enthesal inflammation in the mice. ${ }^{128}$ Scherlock et al identified an enthesal resident T-cell population that express the IL-23 receptor in this mice model and showed that entheseal tissues respond to IL-23 by increasing the expression of IL-17 and Il-22 and of BMP7, which they implicated in the enthesal ossification mechanisms. ${ }^{129}$

UST has been recently shown to demonstrate significant clinical improvement in 20 patients with AS in a prospective, proof of concept clinical trial. ${ }^{130}$

SEC showed, in a small pilot study $(\mathrm{n}=29)$, an ASAS 20 response at week 6 in $60 \%$ of patients with AS compared with $17 \%$ of placebo group. ${ }^{131}$ These data identify the IL-23/Th17 pathway as an important key target in SpAs.

\section{CONCLUSIONS}

Several aspects of bone involvement in RA, SpAs and OA have been highlighted during the last two OsteoRheumatology meetings, held in 2013 and 2014. Although, a number of issues still remain to be clarified, it seems quite clear that in clinical practice, as well as in basic and translational research, there is a need for more knowledge of the interactions between the cartilage, the immune system and the bone. In this context, 'OsteoRheumatology' represents a new discipline that provides a greater insight into this interplay, in order to face the multifactorial and complex issues underlying common and disabling rheumatic diseases.

\section{Author affiliations}

${ }^{1}$ Rheumatology Unit, Department of Medicine-DIMED, University Hospital of Padova, Padova, Italy

${ }^{2}$ Laboratory of Immunorheumatology and Tissue Regeneration/RAMSES,

Rizzoli Orthopedic Research Institute, Bologna, Italy

${ }^{3}$ Bone Clinic, Department of Gerontology and Musculoskeletal Sciences, Galliera Hospital, Genoa, Italy

${ }^{4}$ Department of Internal Medicine, Subdivision of Rheumatology, CAPHRI/ NUTRIM, Maastricht University Medical Centre, Maastricht, The Netherlands \& UHasselt, Belgium

${ }^{5}$ Hospital for Special Surgery and Weill Cornell Medical College, New York, New York, USA

${ }^{6}$ Department of Rheumatology, VU Medical Centre, Amsterdam, The Netherlands

${ }^{7}$ Department of Internal Medicine 3, University of Erlangen-Nuremberg, Erlangen, Germany

${ }^{8}$ Department of Locomotor System, Division of Rheumatology, ASL3 Genovese, Genoa, Italy

Contributors MF, AG, GB and GS took part in review, conception and design. MF and $A G$ participated in drafting the manuscript. MF, AG, PG, SRG, WL, GS and GB critically reviewed the manuscript.

\section{Competing interests None declared.}

Provenance and peer review Commissioned; externally peer reviewed.

Data sharing statement No additional data are available.

Open Access This is an Open Access article distributed in accordance with the Creative Commons Attribution Non Commercial (CC BY-NC 4.0) license, which permits others to distribute, remix, adapt, build upon this work noncommercially, and license their derivative works on different terms, provided the original work is properly cited and the use is non-commercial. See: http:// creativecommons.org/licenses/by-nc/4.0/

\section{REFERENCES}

1. Goldring SR, Goldring MB. Clinical aspects, pathology and pathophysiology of osteoarthritis. J Musculoskelet Neuronal Interact 2006;6:376-8.

2. Loeser RF, Goldring SR, Scanzello CR, et al. Osteoarthritis: a disease of the joint as an organ. Arthritis Rheum 2012;64:1697-707.

3. Berenbaum F. Diabetes-induced osteoarthritis: from a new paradigm to a new phenotype. Ann Rheum Dis 2011;70:1354-6.

4. Roman-Blas JA, Castaneda S, Largo R, et al. Osteoarthritis associated with estrogen deficiency. Arthritis Res Ther 2009;11:241.

5. Zhuo Q, Yang W, Chen J, et al. Metabolic syndrome meets osteoarthritis. Nat Rev Rheumatol 2012;8:729-37.

6. Castaneda S, Largo R, Calvo E, et al. Effects of estrogen deficiency and low bone mineral density on healthy knee cartilage in rabbits. J Orthop Res 2010;28:812-18.

7. Calvo E, Castaneda S, Largo R, et al. Osteoporosis increases the severity of cartilage damage in an experimental model of osteoarthritis in rabbits. Osteoarthritis Cartilage 2007;15:69-77.

8. Buckland-Wright JC, Messent EA, Bingham CO III, et al. A 2 yr longitudinal radiographic study examining the effect of a bisphosphonate (risedronate) upon subchondral bone loss in osteoarthritic knee patients. Rheumatology (Oxford) 2007;46:257-64.

9. Goldring SR, Goldring MB. Bone and cartilage in osteoarthritis: is what's best for one good or bad for the other? Arthritis Res Ther 2010;12:143.

10. Radin EL, Rose RM. Role of subchondral bone in the initiation and progression of cartilage damage. Clin Orthop Relat Res 1986;213:34-40.

11. Sharma AR, Jagga S, Lee SS, et al. Interplay between cartilage and subchondral bone contributing to pathogenesis of osteoarthritis. Int J Mol Sci 2013;14:19805-30. 
12. Castaneda S, Roman-Blas JA, Largo R, et al. Subchondral bone as a key target for osteoarthritis treatment. Biochem Pharmacol 2012;83:315-23

13. Bailey AJ, Mansell JP, Sims TJ, et al. Biochemical and mechanical properties of subchondral bone in osteoarthritis. Biorheology 2004;41:349-58.

14. Wang SX, Laverty S, Dumitriu M, et al. The effects of glucosamine hydrochloride on subchondral bone changes in an animal model of osteoarthritis. Arthritis Rheum 2007;56:1537-48.

15. Roman-Blas JA, Castaneda S, Largo R, et al. An OA phenotype may obtain major benefit from bone-acting agents. Semin Arthritis Rheum 2014:43:421-8.

16. Henrotin Y, Pesesse L, Sanchez C. Subchondral bone and osteoarthritis: biological and cellular aspects. Osteoporos Int 2012;23(Suppl 8):S847-51.

17. Sanchez C, Deberg MA, Bellahcene A, et al. Phenotypic characterization of osteoblasts from the sclerotic zones of osteoarthritic subchondral bone. Arthritis Rheum 2008;58:442-55.

18. Verborgt O, Gibson GJ, Schaffler MB. Loss of osteocyte integrity in association with microdamage and bone remodeling after fatigue in vivo. J Bone Miner Res 2000;15:60-7.

19. Robling AG, Niziolek PJ, Baldridge LA, et al. Mechanical stimulation of bone in vivo reduces osteocyte expression of Sost/ sclerostin. J Biol Chem 2008;283:5866-75.

20. Bellido T, Ali AA, Gubrij I, et al. Chronic elevation of parathyroid hormone in mice reduces expression of sclerostin by osteocytes: a novel mechanism for hormonal control of osteoblastogenesis. Endocrinology 2005;146:4577-83.

21. Chan BY, Fuller ES, Russell AK, et al. Increased chondrocyte sclerostin may protect against cartilage degradation in osteoarthritis. Osteoarthritis Cartilage 2011;19:874-85.

22. Appel H, Ruiz-Heiland G, Listing J, et al. Altered skeletal expression of sclerostin and its link to radiographic progression in ankylosing spondylitis. Arthritis Rheum 2009;60:3257-62.

23. Xiong J, Onal M, Jilka RL, et al. Matrix-embedded cells control osteoclast formation. Nat Med 2011;17:1235-41.

24. Nakashima T, Hayashi M, Fukunaga $T$, et al. Evidence for osteocyte regulation of bone homeostasis through RANKL expression. Nat Med 2011;17:1231-4.

25. Kennedy OD, Herman BC, Laudier DM, et al. Activation of resorption in fatigue-loaded bone involves both apoptosis and active pro-osteoclastogenic signaling by distinct osteocyte populations. Bone 2012;50:1115-22.

26. Moreno-Rubio J, Herrero-Beaumont G, Tardio L, et al. Nonsteroidal antiinflammatory drugs and prostaglandin $\mathrm{E}(2)$ modulate the synthesis of osteoprotegerin and RANKL in the cartilage of patients with severe knee osteoarthritis. Arthritis Rheum 2010;62:478-88.

27. Martinez-Calatrava MJ, Prieto-Potin I, Roman-Blas JA, et al. RANKL synthesized by articular chondrocytes contributes to juxta-articular bone loss in chronic arthritis. Arthritis Res Ther 2012;14:R149.

28. Ko FC, Dragomir C, Plumb DA, et al. In vivo cyclic compression causes cartilage degeneration and subchondral bone changes in mouse tibiae. Arthritis Rheum 2013;65:1569-78.

29. Pesesse L, Sanchez C, Walsh DA, et al. Bone sialoprotein as a potential key factor implicated in the pathophysiology of osteoarthritis. Osteoarthritis Cartilage 2014;22:547-56.

30. Crotti TN, O'Sullivan RP, Shen Z, et al. Bone matrix regulates osteoclast differentiation and annexin A8 gene expression. J Cell Physiol 2011;226:3413-21

31. Hochberg MC, Altman RD, April KT, et al. American College of Rheumatology 2012 recommendations for the use of nonpharmacologic and pharmacologic therapies in osteoarthritis of the hand, hip, and knee. Arthritis Care Res (Hoboken) 2012;64:465-74.

32. Hawker GA, Davis AM, French MR, et al. Development and preliminary psychometric testing of a new OA pain measure-an OARSI/OMERACT initiative. Osteoarthritis Cartilage 2008;16:409-14.

33. Hawker GA, Stewart L, French MR, et al. Understanding the pain experience in hip and knee osteoarthritis-an OARSI/OMERACT initiative. Osteoarthritis Cartilage 2008;16:415-22.

34. Neogi T. The epidemiology and impact of pain in osteoarthritis. Osteoarthritis Cartilage 2013;21:1145-53.

35. Malfait AM, Schnitzer TJ. Towards a mechanism-based approach to pain management in osteoarthritis. Nat Rev Rheumato 2013;9:654-64.

36. Bingel U, Tracey I. Imaging CNS modulation of pain in humans Physiology (Bethesda) 2008;23:371-80.

37. Goldring MB, Marcu KB. Cartilage homeostasis in health and rheumatic diseases. Arthritis Res Ther 2009;11:224.
38. Mease PJ, Hanna S, Frakes EP, et al. Pain mechanisms in osteoarthritis: understanding the role of central pain and current approaches to its treatment. J Rheumatol 2011;38:1546-51.

39. Walsh DA, McWilliams DF, Turley MJ, et al. Angiogenesis and nerve growth factor at the osteochondral junction in rheumatoid arthritis and osteoarthritis. Rheumatology (Oxford) 2010;49:1852-61.

40. Lane NE, Schnitzer TJ, Birbara CA, et al. Tanezumab for the treatment of pain from osteoarthritis of the knee. $N$ Engl J Med 2010;363:1521-31.

41. Schnitzer TJ, Marks JA. A systematic review of the efficacy and general safety of antibodies to NGF in the treatment of OA of the hip or knee. Osteoarthritis Cartilage 2015;23(Suppl 1):S8-17.

42. Seidel MF, Wise BL, Lane NE. Nerve growth factor: an update on the science and therapy. Osteoarthritis Cartilage 2013;21:1223-8.

43. Ogbonna AC, Clark AK, Gentry C, et al. Pain-like behaviour and spinal changes in the monosodium iodoacetate model of osteoarthritis in C57Bl/6 mice. Eur J Pain 2013;17:514-26.

44. Hirsch S, Corradini L, Just S, et al. The CGRP receptor antagonist BIBN4096BS peripherally alleviates inflammatory pain in rats. Pain 2013;154:700-7.

45. Bullock CM, Wookey P, Bennett A, et al. Peripheral calcitonin gene-related peptide receptor activation and mechanical sensitization of the joint in rat models of osteoarthritis pain. Arthritis Rheumatol 2014;66:2188-200.

46. Ashraf S, Mapp PI, Walsh DA. Contributions of angiogenesis to inflammation, joint damage, and pain in a rat model of osteoarthritis. Arthritis Rheum 2011;63:2700-10.

47. Takeshita M, Nakamura J, Ohtori S, et al. Sensory innervation and inflammatory cytokines in hypertrophic synovia associated with pain transmission in osteoarthritis of the hip: a case-control study. Rheumatology (Oxford) 2012;51:1790-5

48. Bullock CM, Kelly S. Calcitonin gene-related peptide receptor antagonists: beyond migraine pain-a possible analgesic strategy for osteoarthritis? Curr Pain Headache Rep 2013;17:375.

49. Baker K, Grainger A, Niu J, et al. Relation of synovitis to knee pain using contrast-enhanced MRIs. Ann Rheum Dis 2010;69:1779-83.

50. Felson DT. Imaging abnormalities that correlate with joint pain. $\mathrm{Br} J$ Sports Med 2011;45:289-91.

51. Hill CL, Gale DG, Chaisson CE, et al. Knee effusions, popliteal cysts, and synovial thickening: association with knee pain in osteoarthritis. J Rheumatol 2001;28:1330-7.

52. Scanzello CR, McKeon B, Swaim BH, et al. Synovial inflammation in patients undergoing arthroscopic meniscectomy: molecular characterization and relationship to symptoms. Arthritis Rheum 2011;63:391-400.

53. Kulkarni B, Bentley DE, Elliott R, et al. Arthritic pain is processed in brain areas concerned with emotions and fear. Arthritis Rheum 2007;56:1345-54.

54. Taljanovic MS, Graham AR, Benjamin JB, et al. Bone marrow edema pattern in advanced hip osteoarthritis: quantitative assessment with magnetic resonance imaging and correlation with clinical examination, radiographic findings, and histopathology. Skeletal Radiol 2008;37:423-31.

55. Yoneda T, Hata K, Nakanishi M, et al. Involvement of acidic microenvironment in the pathophysiology of cancer-associated bone pain. Bone 2011;48:100-5.

56. Davis AJ, Smith TO, Hing CB, et al. Are bisphosphonates effective in the treatment of osteoarthritis pain? A meta-analysis and systematic review. PLOS ONE 2013;8:e72714.

57. Herrero-Beaumont G, Roman-Blas JA. Osteoarthritis: osteoporotic $\mathrm{OA}$ : a reasonable target for bone-acting agents. Nat Rev Rheumatol 2013;9:448-50.

58. Laslett LL, Dore DA, Quinn SJ, et al. Zoledronic acid reduces knee pain and bone marrow lesions over 1 year: a randomised controlled trial. Ann Rheum Dis 2012;71:1322-8.

59. Moreau M, Rialland P, Pelletier JP, et al. Tiludronate treatment improves structural changes and symptoms of osteoarthritis in the canine anterior cruciate ligament model. Arthritis Res Ther 2011;13:R98

60. Walsh DA, Chapman V. Bisphosphonates for osteoarthritis. Arthritis Res Ther 2011;13:128.

61. Bruyere O, Reginster JY, Bellamy N, et al. Clinically meaningful effect of strontium ranelate on symptoms in knee osteoarthritis: a responder analysis. Rheumatology (Oxford) 2014;53:1457-64

62. Reginster JY. Efficacy and safety of strontium ranelate in the treatment of knee osteoarthritis: results of a double-blind randomised, placebo-controlled trial. Ann Rheum Dis 2014;73:e8.

63. Pelletier JP, Roubille C, Raynauld JP, et al. Disease-modifying effect of strontium ranelate in a subset of patients from the Phase III knee osteoarthritis study SEKOIA using quantitative MRI: reduction in bone 
marrow lesions protects against cartilage loss. Ann Rheum Dis 2015;74:422-9.

64. Bolland MJ, Grey A. A comparison of adverse event and fracture efficacy data for strontium ranelate in regulatory documents and the publication record. BMJ Open 2014;4:e005787.

65. Bondeson J. Are we moving in the right direction with osteoarthritis drug discovery? Expert Opin Ther Targets 2011;15:1355-68.

66. Verbruggen G, Wittoek R, Vander Cruyssen B, et al. Tumour necrosis factor blockade for the treatment of erosive osteoarthritis of the interphalangeal finger joints: a double blind, randomised trial on structure modification. Ann Rheum Dis 2012;71:891-8.

67. Maksymowych WP, Russell AS, Chiu P, et al. Targeting tumour necrosis factor alleviates signs and symptoms of inflammatory osteoarthritis of the knee. Arthritis Res Ther 2012;14:R206.

68. Hellio le Graverand MP, Clemmer RS, Redifer P, et al. A 2-year randomised, double-blind, placebo-controlled, multicentre study of oral selective iNOS inhibitor, cindunistat (SD-6010), in patients with symptomatic osteoarthritis of the knee. Ann Rheum Dis 2013;72:187-95.

69. Chevalier X, Goupille P, Beaulieu AD, et al. Intraarticular injection of anakinra in osteoarthritis of the knee: a multicenter, randomized, double-blind, placebo-controlled study. Arthritis Rheum 2009;61:344-52.

70. Cohen SB, Proudman S, Kivitz AJ, et al. A randomized double-blind study of AMG 108 (a fully human monoclonal antibody to IL-1R1) in patients with osteoarthritis of the knee. Arthritis Res Ther 2011;13:R125.

71. Brown C, Toth A, Magnussen R. Clinical benefits of intra-articular anakinra for persistent knee effusion. J Knee Surg $2011 ; 24: 61-5$

72. Kraus VB, Birmingham J, Stabler TV, et al. Effects of intraarticular IL1-Ra for acute anterior cruciate ligament knee injury: a randomized controlled pilot trial (NCT00332254). Osteoarthritis Cartilage 2012;20:271-8.

73. Gravallese EM, Harada Y, Wang JT, et al. Identification of cell types responsible for bone resorption in rheumatoid arthritis and juvenile rheumatoid arthritis. Am J Pathol 1998;152:943-51.

74. Haynes DR, Crotti TN, Loric M, et al. Osteoprotegerin and receptor activator of nuclear factor kappaB ligand (RANKL) regulate osteoclast formation by cells in the human rheumatoid arthritic joint. Rheumatology (Oxford) 2001;40:623-30.

75. Itonaga I, Fujikawa $\mathrm{Y}$, Sabokbar A, et al. Rheumatoid arthritis synovial macrophage-osteoclast differentiation is osteoprotegerin ligand-dependent. J Pathol 2000;192:97-104.

76. Lubberts E, Oppers-Walgreen B, Pettit AR, et al. Increase in expression of receptor activator of nuclear factor kappaB at sites of bone erosion correlates with progression of inflammation in evolving collagen-induced arthritis. Arthritis Rheum 2002;46:3055-64.

77. Suzuki Y, Tsutsumi Y, Nakagawa M, et al. Osteoclast-like cells in an in vitro model of bone destruction by rheumatoid synovium. Rheumatology (Oxford) 2001;40:673-82.

78. Takayanagi $\mathrm{H}$, lizuka $\mathrm{H}$, Juji $\mathrm{T}$, et al. Involvement of receptor activator of nuclear factor kappaB ligand/osteoclast differentiation factor in osteoclastogenesis from synoviocytes in rheumatoid arthritis. Arthritis Rheum 2000;43:259-69.

79. Pettit AR, Walsh NC, Manning C, et al. RANKL protein is expressed at the pannus-bone interface at sites of articular bone erosion in rheumatoid arthritis. Rheumatology (Oxford) 2006;45:1068-76.

80. Pettit AR, Ji H, von Stechow D, et al. TRANCE/RANKL knockout mice are protected from bone erosion in a serum transfer model of arthritis. Am J Pathol 2001;159:1689-99.

81. Li P, Schwarz EM, O'Keefe RJ, et al. RANK signaling is not required for TNFalpha-mediated increase in CD11(hi) osteoclast precursors but is essential for mature osteoclast formation in TNFalpha-mediated inflammatory arthritis. J Bone Miner Res 2004:19:207-13.

82. Redlich K, Hayer S, Ricci R, et al. Osteoclasts are essential for TNF-alpha-mediated joint destruction. J Clin Invest 2002;110:1419-27.

83. Matzelle MM, Gallant MA, Condon KW, et al. Resolution of inflammation induces osteoblast function and regulates the Wnt signaling pathway. Arthritis Rheum 2012;64:1540-50.

84. Moon RT, Kohn AD, De Ferrari GV, et al. WNT and beta-catenin signalling: diseases and therapies. Nat Rev Genet 2004;5:691-701.

85. Li C, Ominsky MS, Tan HL, et al. Increased callus mass and enhanced strength during fracture healing in mice lacking the sclerostin gene. Bone 2011;49:1178-85.
86. Li X, Ominsky MS, Warmington KS, et al. Increased bone formation and bone mass induced by sclerostin antibody is not affected by pretreatment or cotreatment with alendronate in osteopenic, ovariectomized rats. Endocrinology 2011;152:3312-22.

87. Marenzana M, Greenslade K, Eddleston A, et al. Sclerostin antibody treatment enhances bone strength but does not prevent growth retardation in young mice treated with dexamethasone. Arthritis Rheum 2011;63:2385-95.

88. Diarra D, Stolina M, Polzer K, et al. Dickkopf-1 is a master regulator of joint remodeling. Nat Med 2007;13:156-63.

89. Berglin E, Padyukov L, Sundin U, et al. A combination of autoantibodies to cyclic citrullinated peptide (CCP) and HLA-DRB1 locus antigens is strongly associated with future onset of rheumatoid arthritis. Arthritis Res Ther 2004;6:R303-8.

90. Nielen MM, van Schaardenburg D, Reesink HW, et al. Specific autoantibodies precede the symptoms of rheumatoid arthritis: a study of serial measurements in blood donors. Arthritis Rheum 2004;50:380-6.

91. Rantapaa-Dahlqvist S, de Jong BA, Berglin E, et al. Antibodies against cyclic citrullinated peptide and $\lg A$ rheumatoid factor predict the development of rheumatoid arthritis. Arthritis Rheum 2003;48:2741-9

92. Mclnnes IB, Schett $\mathrm{G}$. The pathogenesis of rheumatoid arthritis. N Engl J Med 2011;365:2205-19.

93. Geusens P, Lems WF. Osteoimmunology and osteoporosis. Arthritis Res Ther 2011;13:242.

94. Goldring SR, Purdue PE, Crotti TN, et al. Bone remodelling in inflammatory arthritis. Ann Rheum Dis 2013;72(Suppl 2):ii52-5.

95. Haugeberg G, Uhlig T, Falch JA, et al. Bone mineral density and frequency of osteoporosis in female patients with rheumatoid arthritis: results from 394 patients in the Oslo County Rheumatoid Arthritis register. Arthritis Rheum 2000;43:522-30.

96. Kocijan R, Finzel S, Englbrecht M, et al. Decreased quantity and quality of the periarticular and nonperiarticular bone in patients with rheumatoid arthritis: a cross-sectional HR-pQCT study. J Bone Miner Res 2014;29:1005-14.

97. Krieckaert CL, Nurmohamed MT, Wolbink G, et al. Changes in bone mineral density during long-term treatment with adalimumab in patients with rheumatoid arthritis: a cohort study. Rheumatology (Oxford) 2013;52:547-53.

98. Sinigaglia L, Varenna M, Girasole G, et al. Epidemiology of osteoporosis in rheumatic diseases. Rheum Dis Clin North Am 2006;32:631-58

99. Kleyer A, Finzel S, Rech J, et al. Bone loss before the clinical onset of rheumatoid arthritis in subjects with anticitrullinated protein antibodies. Ann Rheum Dis 2014;73:854-60.

100. de Rooy DP, Kalvesten J, Huizinga TW, et al. Loss of metacarpal bone density predicts RA development in recent-onset arthritis. Rheumatology (Oxford) 2012;51:1037-41.

101. Guler-Yuksel M, Bijsterbosch J, Goekoop-Ruiterman YP, et al. Bone mineral density in patients with recently diagnosed, active rheumatoid arthritis. Ann Rheum Dis 2007;66:1508-12.

102. van Schaardenburg D, Nielen MM, Lems WF, et al. Bone metabolism is altered in preclinical rheumatoid arthritis. Ann Rheum Dis 2011;70:1173-4.

103. Kocijan R, Harre U, Schett G. ACPA and bone loss in rheumatoid arthritis. Curr Rheumatol Rep 2013;15:366.

104. Harre $U$, Georgess D, Bang $\mathrm{H}$, et al. Induction of osteoclastogenesis and bone loss by human autoantibodies against citrullinated vimentin. J Clin Invest 2012;122:1791-802.

105. Hetland ML, Ejbjerg B, Horslev-Petersen K, et al. MRI bone oedema is the strongest predictor of subsequent radiographic progression in early rheumatoid arthritis. Results from a 2-year randomised controlled trial (CIMESTRA). Ann Rheum Dis 2009;68:384-90.

106. Takayanagi H. Osteoimmunology: shared mechanisms and crosstalk between the immune and bone systems. Nat Rev Immunol 2007;7:292-304.

107. Schett G, Stolina M, Bolon B, et al. Analysis of the kinetics of osteoclastogenesis in arthritic rats. Arthritis Rheum 2005;52:3192-201.

108. Horowitz M, Vignery A, Gershon RK, et al. Thymus-derived lymphocytes and their interactions with macrophages are required for the production of osteoclast-activating factor in the mouse. Proc Natl Acad Sci USA 1984;81:2181-5.

109. Gravallese EM, Manning C, Tsay A, et al. Synovial tissue in rheumatoid arthritis is a source of osteoclast differentiation factor. Arthritis Rheum 2000;43:250-8.

110. Shigeyama Y, Pap T, Kunzler P, et al. Expression of osteoclast differentiation factor in rheumatoid arthritis. Arthritis Rheum 2000;43:2523-30. 
111. McClung MR, Lewiecki EM, Cohen SB, et al. Denosumab in postmenopausal women with low bone mineral density. $N$ Engl $J$ Med 2006;354:821-31.

112. Cohen SB, Dore RK, Lane NE, et al. Denosumab treatment effects on structural damage, bone mineral density, and bone turnover in rheumatoid arthritis: a twelve-month, multicenter, randomized, double-blind, placebo-controlled, phase II clinical trial. Arthritis Rheum 2008;58:1299-309.

113. Deodhar A, Dore RK, Mandel D, et al. Denosumab-mediated increase in hand bone mineral density associated with decreased progression of bone erosion in rheumatoid arthritis patients. Arthritis Care Res (Hoboken) 2010;62:569-74.

114. Mclnnes IB, Schett G. Cytokines in the pathogenesis of rheumatoid arthritis. Nat Rev Immunol 2007;7:429-42.

115. Hayer S, Steiner G, Gortz B, et al. CD44 is a determinant of inflammatory bone loss. J Exp Med 2005;201:903-14.

116. Lam J, Takeshita S, Barker JE, et al. TNF-alpha induces osteoclastogenesis by direct stimulation of macrophages exposed to permissive levels of RANK ligand. J Clin Invest 2000;106:1481-8.

117. Schett G, Gravallese E. Bone erosion in rheumatoid arthritis: mechanisms, diagnosis and treatment. Nat Rev Rheumatol 2012;8:656-64.

118. Walsh NC, Reinwald S, Manning CA, et al. Osteoblast function is compromised at sites of focal bone erosion in inflammatory arthritis. J Bone Miner Res 2009;24:1572-85.

119. Goldring SR. Osteoimmunology and bone homeostasis: relevance to spondyloarthritis. Curr Rheumatol Rep 2013;15:342.

120. Lories RJ, Luyten FP, de Vlam K. Progress in spondylarthritis. Mechanisms of new bone formation in spondyloarthritis. Arthritis Res Ther 2009;11:221.

121. Lories RJ, Schett G. Pathophysiology of new bone formation and ankylosis in spondyloarthritis. Rheum Dis Clin North Am 2012;38:555-67.
122. Lories RJ, Derese I, Luyten FP. Modulation of bone morphogenetic protein signaling inhibits the onset and progression of ankylosing enthesitis. J Clin Invest 2005;115:1571-9.

123. Uderhardt S, Diarra D, Katzenbeisser J, et al. Blockade of Dickkop (DKK)-1 induces fusion of sacroiliac joints. Ann Rheum Dis 2010;69:592-7.

124. Louie GH, Ward MM. Measurement and treatment of radiographic progression in ankylosing spondylitis: lessons learned from observational studies and clinical trials. Curr Opin Rheumatol 2014;26:145-50.

125. Baraliakos X, Haibel H, Listing J, et al. Continuous long-term anti-TNF therapy does not lead to an increase in the rate of new bone formation over 8 years in patients with ankylosing spondylitis. Ann Rheum Dis 2014;73:710-15.

126. Haroon $\mathrm{N}$, Inman $\mathrm{RD}$, Learch $\mathrm{TJ}$, et al. The impact of tumor necrosis factor alpha inhibitors on radiographic progression in ankylosing spondylitis. Arthritis Rheum 2013;65:2645-54.

127. Braun J, Kiltz U, Heldmann F, et al. Emerging drugs for the treatment of axial and peripheral spondyloarthritis. Expert Opin Emerg Drugs 2015;20:1-14.

128. Sherlock JP, Joyce-Shaikh B, Turner SP, et al. IL-23 induces spondyloarthropathy by acting on ROR-gammat+ CD3+CD4-CD8entheseal resident T cells. Nat Med 2012;18:1069-76.

129. Wendling D, Guillot X, Prati C. The IL-23/Th 17 pathway in spondyloarthritis: the royal road? Joint Bone Spine 2015;82:1-4.

130. Poddubnyy D, Hermann KG, Callhoff J, et al. Ustekinumab for the treatment of patients with active ankylosing spondylitis: results of a 28-week, prospective, open-label, proof-of-concept study (TOPAS). Ann Rheum Dis 2014;73:817-23.

131. Baeten D, Baraliakos X, Braun J, et al. Anti-interleukin-17A monoclonal antibody secukinumab in treatment of ankylosing spondylitis: a randomised, double-blind, placebo-controlled trial. Lancet 2013;382:1705-13. 